Nepal Journal of Multidisciplinary Research (NJMR)

Vol. 4, No. 4, December 2021. Pages: 70-74

ISSN: 2645-8470 (Print), ISSN: 2705-4691 (Online)

DOI: $\underline{\text { https://doi.org/10.3126/njmr.v4i4.43218 }}$

\title{
Exploring the Views and Dietary Practices of Elderly Nepalese Citizen: A Qualitative Study
}

\author{
Maya Nath Ghimire, PhD \\ Nepal philosophical Research Center, Kathmandu, Nepal \\ mayanathghimire@gmail.com
}

Received: November 22, 2021; Revised \& Accepted: December 29, 2021

(C) Copyright: Ghimire (2021).

(c) (1) \&

International License.

\begin{abstract}
One of the main causes of morbidity and mortality of old-age people is malnutrition. We have very less studies on Nepalese elder citizen's food practice. The aim of this study is to explore food practice of Nepalese elder citizen. A qualitative study was performed using in-depth interviews.The study was conducted in two districts of Nepal: Lamjung and Chitawan. All the respondents were 71-91 years elder citizen. The findings show that almost adolescents' normal wakeup time is 4-5 AM. They consume tea, biscuits, milk, chapatti, beaten rice as breakfast; pulse, vegetable, milk, butter and weekly meat with normal food for lunch and dinner. Generally, they consume gravy vegetable curry with rice and chapatti. However, they prefer vegetarian soft and hot food.
\end{abstract}

Keywords: elder people, food practice, food choice

\section{Introduction}

Nepal has distinctly two groups: rural and urban, in case of food consumption pattern. Moreover, industrialization and urbanization has changed consumption pattern. The rural setting has its own pattern of food consumption based on availability of food according to season, find in the consumers' own farmland (Neupane, 219; Neupane, 2018).

Chalobol Chalermsri and friends wrote in the context of Thailand that they had experienced a rapid increase in its elderly population. There are many unfavorable health outcomes among elderly people are associated with nutrition. Nutrition in elderly people is affected by physical, mental, and social factors. This study explored the food choices and dietary practices among community-dwelling elderly people in Thailand from the perspective of both caregivers and the 


\title{
Nepal Journal of Multidisciplinary Research (NJMR)
}

\author{
Vol. 4, No. 4, December 2021. Pages: 70-74 \\ ISSN: 2645-8470 (Print), ISSN: 2705-4691 (Online) \\ DOI: https://doi.org/10.3126/njmr.v4i4.43218
}

elderly people themselves (Chalermsri, Wees, Ziaei, Ekström, Muangpaisan, \& Rahman, 2020, p. 1) .

According to Zumin Shi and friends, they have examined the association between food habits, lifestyle factors and all-cause mortality in the oldest age using data from the Chinese Longitudinal Healthy Longevity Survey (CLHLS). In 1998/99, 8959 participants aged 80 years and older took part in the baseline survey. Follow-up surveys were conducted every two to three years until 2011 (Placeholder2) (Shi, Zhang, Byles, Martin, Avery, \& Taylor, 2015, p. 7562).

Professor Kourkouta Lambrini writes the population of people living in the Elderly has increased significantly in recent decades. In Europe, $1 / 4$ of the population is aged over 60 years old. Although the life expectancy has increased, mainly due to improved diagnostic and therapeutic approach of the diseases is not known whether the added years are of good quality, i.e. if these people living without significant health problems which can greatly affect their lifestyle (L, P., A, \& Ch., 2016, p. 155).

Helldan, T. Lallukka and his friend wrote Numerous factors are involved in determining eating ha-bits including gender, marital tatus and household compo-sition, socio-economic position, income, physical activity, smoking, body mass index (BMI) and health but the influ-ence of these factors on eating habits has received little consideration among older adults (Helldan T. L., 2012, p. 582).

Maya Nath Ghimire wrote the multi sector indicators work in bidirectional pathways; meaning they are contributors to poor nutrition and food insecurity and can also be their manifestations. Most of the development indicators continue to have wide disparities by region, sub-region and urban versus rural areas in Nepal (Ghimire, 2018, p. 172).

\section{Objective}

The main objective of this study is to explore status of Nepalese Elders' food choice from Lamjung and Chitawan, Nepal.

\section{Methodology}

The research was an explorative based on qualitative method. The study was conducted in Lamjung and Chitawan districts, Nepal. Eligible participants were 71-91 years elder male and female. The data were collected through in-depth interviews with semi-structure questionnaire. While talking about the philosophical foundation, philosophical stance guides the researcher about the way in which the data about a phenomenon were gathered, analyzed and report prepared. So research paradigm in a qualitative research acts as the guide of any research since it talks about food trend of their life. It also deals with the researchers and their existence as well. Research paradigm is a collection of belief, which guides any researcher to decide what can be studied and how results can be interpreted. I decided to use the interpretive and critical paradigm for this research work. According to Cohen and Mahion (L., 2007, p. 638), interpretive are concerned with understanding the subjective world of the human experience by interviewing or 


\title{
Nepal Journal of Multidisciplinary Research (NJMR)
}

\author{
Vol. 4, No. 4, December 2021. Pages: 70-74 \\ ISSN: 2645-8470 (Print), ISSN: 2705-4691 (Online) \\ DOI: $\underline{\text { https://doi.org/10.3126/njmr.v4i4.43218 }}$
}

observing the participants. This paradigm gives importance to subjective relationship between the researcher and subject. It also helps the researchers to conduct this study in natural environment where the female participation is practiced. The study had a small sample size, but did reach saturation within 20 respondents, which is paramount in qualitative research.Out of 20 respondents, 13 were from Lamjung and 7 from Chitawan. Apply face to face interview was used at data collection. Collected data was complied, analyzed and prepare research report.

\section{Result \& Discussion}

Respondents from rural area of Lamjung and Chitawan districts of Nepal participated in an individual level in-depth interview in the following areas. Respondents' answers were concised and prepared report accordingly.

\subsection{General information}

The age of respondents varied from $71-91$ years old. The respondents were 71-85 years from Lamjung and 73-91 years old from Chitawan. Among them 10 were female and 10 were male.

\subsection{Normal wakeup time and food items eat during breakfast, lunch, snacks and dinner:}

The respondents were asked normal wakeup time, breakfast items, lunch time and items and dinner time and items.

The respondents' wakeup time varied from 4.00 to 7 AM.

'Because of old age, I wake up earlier than crow's caw. Then go to toilet. Come back. Cannot sleep. Roll this side and that side, now become morning. \#Participant 7'.

'My little grandson does not sleep until midnight. He makes noise. He runs one to another room. I cannot sleep till he sleeps. So in the morning I get up late like my grandchild, hahahha. \#Participant 14'.

'I have a buffalo and a calf. The calf wants feeding early morning. Then it starts bleating from 3 AM. So I get up in about 4 AM. This calf needs its mother's milk. This calf is also like our children. We have to satisfy it, isn't it? \#Participant 3'.

'My daughter in-law teaches in the campus. She gets up early in the morning. She has 3 years old son. He also gets up with his mother. My son is in Kathmandu. He works in office. So, I have to look after this little boy, boy is active. Because of this, I get up early in the morning. But, I have got time pass. Sometimes it is difficult when I am not feeling well. He demands to go round this village. I feel too much tired. Ya, because of this work I feel tired and sleep early. Life is like this, happy and sorrow. Play of god. \#Participant 19'.

The respondents were also asked about their breakfast. They have different breakfast culture. 


\title{
Nepal Journal of Multidisciplinary Research (NJMR)
}

\author{
Vol. 4, No. 4, December 2021. Pages: 70-74 \\ ISSN: 2645-8470 (Print), ISSN: 2705-4691 (Online) \\ DOI: $\underline{\text { https://doi.org/10.3126/njmr.v4i4.43218 }}$
}

'I get up early in the morning. I prefer black tea. I prepare tea myself. In the morning I take tea more than four times. I take tea frequently. That's why my granddaughter call me 'Chiya Budhi' (Tea Grandpa). Her mother taught her to say so, hahahaha. \#Participant 2'.

'I have to get up early to feed my calf. I milk buffalo. Boil it. In my family I become the first to take breakfast. Everyday I drink a glass of milk. Then after I go to local tea shop. But I don't drink tea there. Every day I go to tea shop, you know why? Hahaha, just to listen people's gossips. \#Participant3'.

Similarly, some of the respondents replied that they take tea and biscuits, milk and chapatti, tea and beaten rice, etc. One female replied nothing to eat in the morning.

The respondents were also asked about their lunch and dinner. They take lunch from $9 \mathrm{Am}$ to 12 noon. They take snacks from 2 to 5 PM, and dinner time is from 6 to 9 PM.Mostly in the lunch and dinner, they consume rice, gravy vegetable curry. Some of them consume rice, lintel and curry. Occasionally, they consume meat. Very few of them consume balance diet. As snacks, they consumet Tea, Milk, Biscuits, chapatti, fried maize with soya bean.

\subsection{All the consume foods are self-products or purchase}

Respondents replied 30\% self-product and 70\% purchased. These purchase items are rice, pulse, vegetables and fruits, milk.

\subsection{The favorite foods items of elder citizens}

The elder citizens' favorite foods are pulse, rice, milk, butter milk, chapatti, curd, Jeri, meat, fruits (orange). Elder citizen like banana mixed with rice and milk. Almost elder citizen like vegetarian food.

\subsection{Different between 30 - 40 years before and now}

30-40 years before: They used to consume pulse, Rice, vegetable, milk, Fish, Meat (especially in festival), sour \& green chilly food, Colacassia with green Leaf, maize rice and millet (parries \& bread), fried maize with fried soya bean, nettle soup, Fiddlehead (wild Neguro in Nepali found in forest).

Now: They consume pulse, rice, chapatti, gravy vegetable, milk, vegetarian and nonvegetarian food items, daily one spoon honey. Now we have needed hot and simple food in time.

\subsection{Nutritious food for elder age}

Pulse, rice and vegetables, milk, maize rice, millet (Dhindo \& millet bread), nettle are best for elder age food. Organic food, vegetable and fruits, soft and hot foods are best for elder age. 


\section{Nepal Journal of Multidisciplinary Research (NJMR) \\ Vol. 4, No. 4, December 2021. Pages: 70-74 \\ ISSN: 2645-8470 (Print), ISSN: 2705-4691 (Online) \\ DOI: $\underline{\text { https://doi.org/10.3126/njmr.v4i4.43218 }}$}

\subsection{Teenage consume food good or not}

All the respondents replied that present food habit of teenage is not good because they consume junk food (Coke, Panipuri, noodles/chowmin, Mo:mo, bottle and plastic packed). These foods are not good for health. All the market readymade food used chemical for make tasty which are harmful for human health. One respondent replied teenagers eat food, I have no comment, and they like they eat it's their right. Young use drug and hasis (ganja) it is a big problem seeing in society.

\section{Conclusion}

This qualitative study shows that rural setting Nepalese elder citizen's food culture is almost similar. They are aware of organic food consumption. They used to consume maize, rice and millet dhindo (pores and bread), nettle soup, etc. which were purely local production. However, present food supply is mostly dependent upon out sourcing. They prefer vegetarian, soft and hot food in elder age.

\section{References}

Chalermsri, C., Wees, S. H., Ziaei, S., Ekström, E.-C., Muangpaisan, W., \& Rahman, S. M. (2020). Exploring the Experience and Determinants of the Food Choices and Eating Practices of Elderly Thai People: A Qualitative Study. MDPI, 1-15.

Ghimire, M. N. (2018, April 23). Food security practices of Chepang community of Nepal. International Journal of Applied Research, 4(5), 172-177.

Helldan, T. L. (2012). Changes in healthy food habits after transition to old age. Eur J Public Health, 22(4), 582-586.

Helldan, T. L. (2012). Changes in healthy food habits after transition to old age. Eur J Public Health, 582586.

L, K., P., O., A, M., \& Ch., I. (2016, December). Nutritional habits in the elderly. Prog Health Sci, 154-159.

L., C. \&. (2007). Research method in education. British Journal of Education Studies, 55(4), 14-18.

Neupane, D. (2018). Food Choice Motives of Guardians of Trilok Academy Kathmandu with reference to Gender. Nepal Journal of Multidisciplinary Research (NJMR), 1(1), 73-81.

Neupane, D. (219). Food choice motives of adults from Kathmandu city. International Journal of Applied Research, 5(3), 182-185.

Shi, Z., Zhang, T., Byles, J., Martin, S., Avery, J. C., \& Taylor, A. W. (2015). Food Habits, Lifestyle Factors and Mortality among Oldest Old Chinese: The Chinese Longitudinal Healthy Longevity Survey (CLHLS). Nutrients, 7562-7579. 